\title{
Archéopages
}

Archéopages

Archéologie et société

$35 \mid 10 / 2012$

Vivre avec les bêtes

\section{Bergerie et poulailler dans un souterrain médiéval. «Le Bois d'Ormes » dans le Loiret}

Laurent Fournier

\section{(2) OpenEdition}

1 Journals

Édition électronique

URL : https://journals.openedition.org/archeopages/302

DOI : 10.4000/archeopages.302

ISSN : 2269-9872

Éditeur

INRAP - Institut national de recherches archéologiques préventives

Édition imprimée

Date de publication : 1 mai 2013

Pagination : $52-59$

ISSN : 1622-8545

\section{Référence électronique}

Laurent Fournier, «Bergerie et poulailler dans un souterrain médiéval. «Le Bois d'Ormes » dans le Loiret », Archéopages [En ligne], 35| 10/2012, mis en ligne le 01 octobre 2014, consulté le 21 janvier 2022. URL : http://journals.openedition.org/archeopages/302 ; DOI : https://doi.org/10.4000/ archeopages.302 


\title{
Bergerie et poulailler dans \\ un souterrain médiéval \\ «Le Bois d'Ormes » dans le Loiret
}

\author{
Laurent Fournier ${ }^{\text {nrrap }}$
}

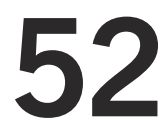

1. Par la société Axis Conseils.
C'est au sein des vestiges de la dernière phase d'occupation $\left(\mathrm{XII}^{\mathrm{e}}\right.$-XIII ${ }^{\mathrm{e}} \mathrm{s}$.) du site fouillé à Ormes qu'une structure souterraine a été découverte (Fournier, 2011) [ill. 1]. Les premiers indices ont été relevés en deux points de la partie sud-ouest de la zone de fouille. Le premier indice est apparu au cours du diagnostic (Rivoire, Liévaux, 2009) : un élément, initialement interprété comme une tranchée de récupération d'un mur situé dans l'extrémité orientale du sondage, a finalement été identifié comme un escalier d'au moins sept degrés [ill. 3]. Les marches sont réalisées dans la couche des sables et argiles de l'Orléanais déposée sur la marne. La présence d'une couche sur les premières marches permet d'envisager l'existence d'un aménagement en bois. La largeur de cet escalier est comprise entre $0,75 \mathrm{~m}$ pour le tronçon septentrional et $0,85 \mathrm{~m}$ pour la partie méridionale au-delà du coude. Le second indice d'une structure souterraine a été découvert à $6 \mathrm{~m}$ au sud-est de l'escalier. Il s'agit d'un puits (F.1-274), grossièrement parementé dans la partie occidentale de son creusement. Il a été partiellement fouillé [ill. 4]. Ces observations ont rapidement conduit à la réalisation d'un sondage mécanique entre l'escalier et le puits. La suite de l'accès présentait de brusques changements d'orientation, décrivant ainsi une suite de chicanes. Ce type d'aménagement est commun dans les souterrains refuges et vise à sécuriser l'accès aux parties souterraines en permettant une défense plus aisée de l'entrée. Au débouché de cet escalier est apparue une galerie de taille modeste desservant des pièces, dont certaines reliées par des boyaux. Après vérification de l'état sanitaire de l'ensemble excavé, la fouille a pu reprendre et un relevé en trois dimensions a été effectué ${ }^{\mathbf{1}}$ [ill. 2].
Description des espaces aériens et souterrains

L'escalier était couvert par un plancher reposant sur des étais. Ceux-ci se trouvaient installés dans des aménagements réalisés dans ses parois [ill. 5]. Lescalier permettant l'accès du souterrain du site médiéval de Pensol « Beaulieu » (Haute-Vienne) présente le même type d'aménagements (Conte, Gauthier, 1985, p. 217). À Ormes, l'accès à la partie souterraine est condamné par une porte dont le dormant est également ancré dans une feuillure creusée dans la paroi de l'escalier. La circulation entre les différents espaces souterrains est assurée au moyen d'une galerie longue de 3,20 $\mathrm{m}$ et haute de $1,55 \mathrm{~m}$. Elle s'interrompt au sud-est contre une maçonnerie grossière condamnant le creusement du puits F.1-274.

Au niveau de l'entrée de la galerie, sur la droite, un silo a été creusé dans la paroi. De forme ovoïde, sa hauteur totale atteint 2,10 $\mathrm{m}$ et son diamètre maximum est de 1,60 m. Son creusement est plus profond de 1,30 $\mathrm{m}$ par rapport au niveau de circulation du couloir.

Lors du dégagement de la galerie principale, un boyau ouvert dans sa paroi nord-ouest a été mis au jour. Son tracé est initialement perpendiculaire à la paroi de la galerie principale sur une distance de o,8o m, puis il oblique et longe ce dernier sur une distance de $1,50 \mathrm{~m}$. La largeur de ce boyau varie d'ouest en est de $0,47 \mathrm{~m}$ à $0,50 \mathrm{~m}$ pour une hauteur constante de $0,40 \mathrm{~m}$. Il débouche au sudest dans une cavité de taille modeste (pièce 1 ) : elle est large de 1,50 $\mathrm{m}$, longue de 2,30 $\mathrm{m}$ pour une hauteur de voûte de 1,50 m. À l'origine, cette pièce communiquait également avec le puits F.1-274, mais ce passage fut condamné par une maçonnerie grossière, similaire à celle découverte à l'extrémité sud-est de la galerie principale. Celle-ci ouvre, à son extrémité sud-est, sur deux pièces en enfilade. 


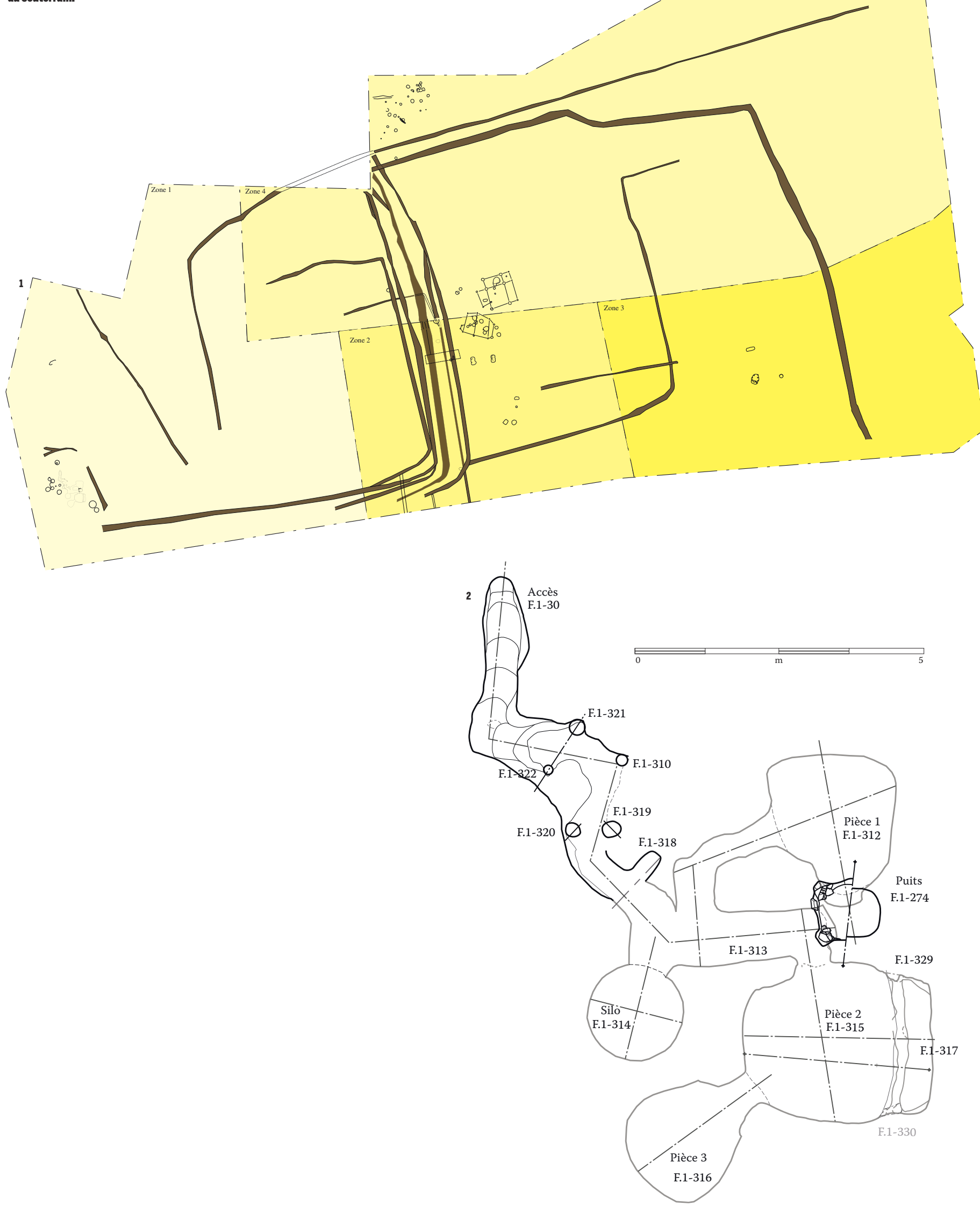




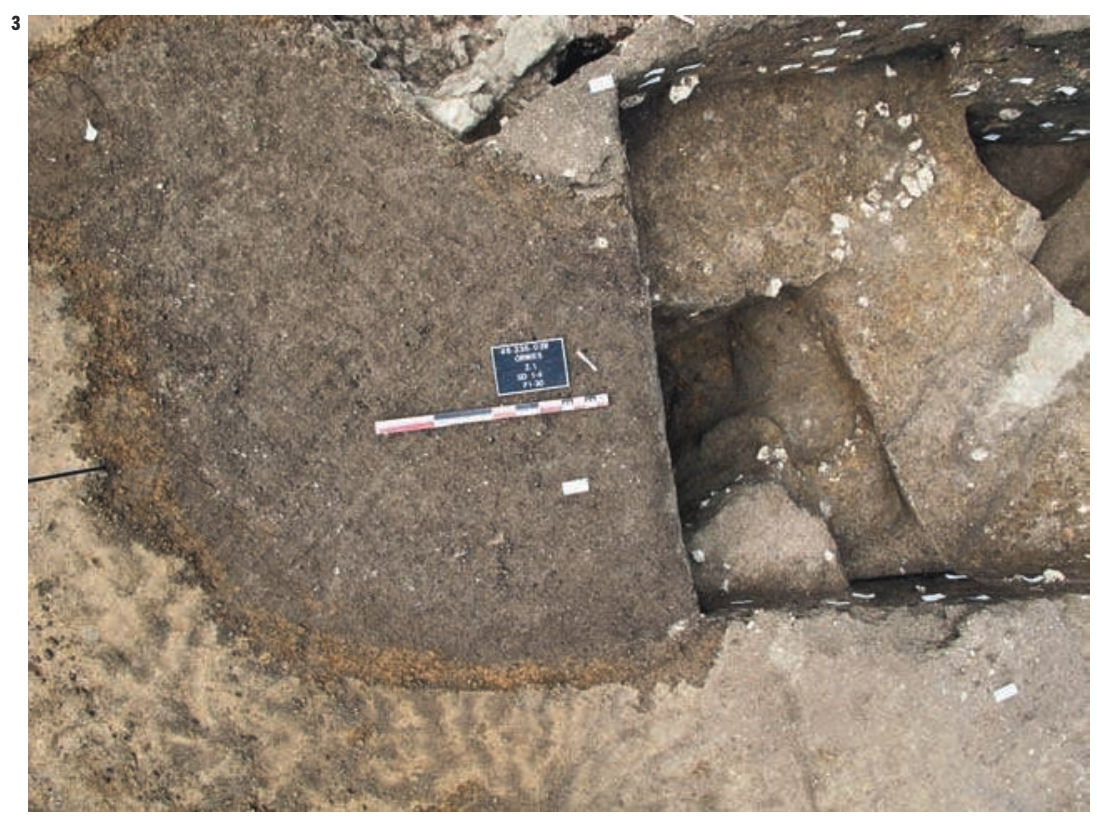

3.Vue de l'escalier

d'accès de la structure

souterraine.

4. Vue du puits d'accès

(F.1-274).

5. Vue des ancrages

des poteaux supportant

la couverture de l'accès

creusé dans les parois

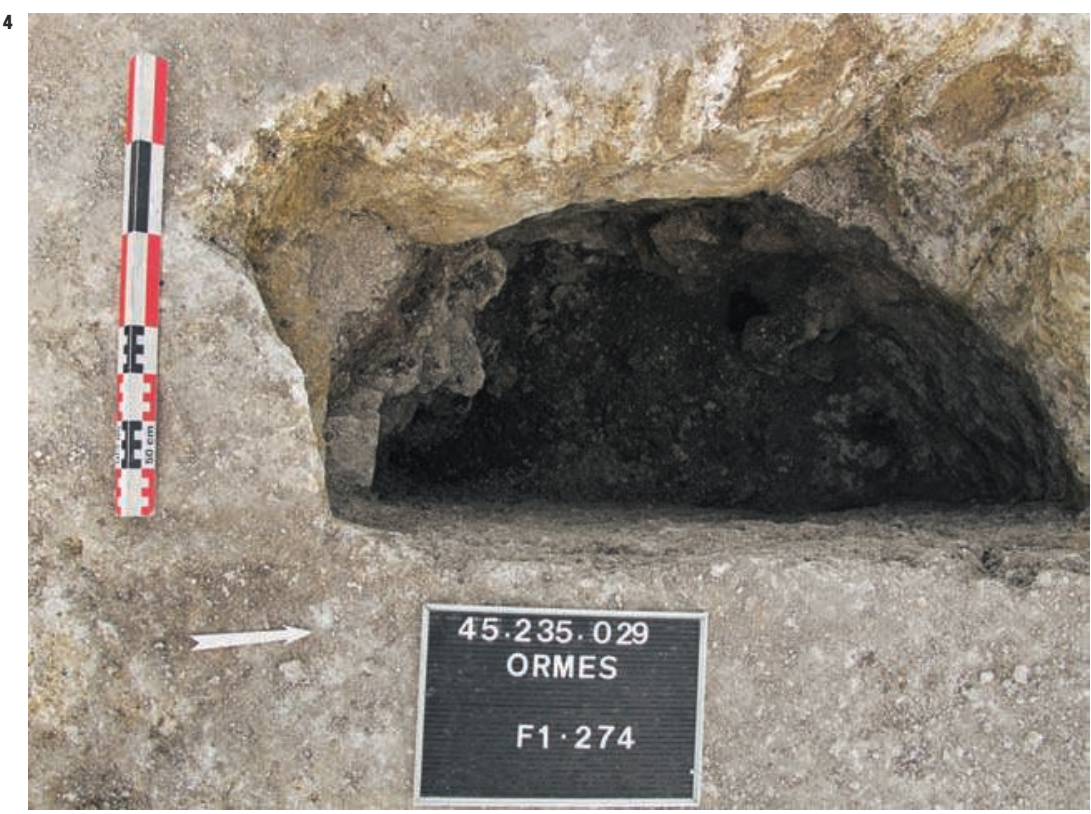

de l'escalier d'accès.

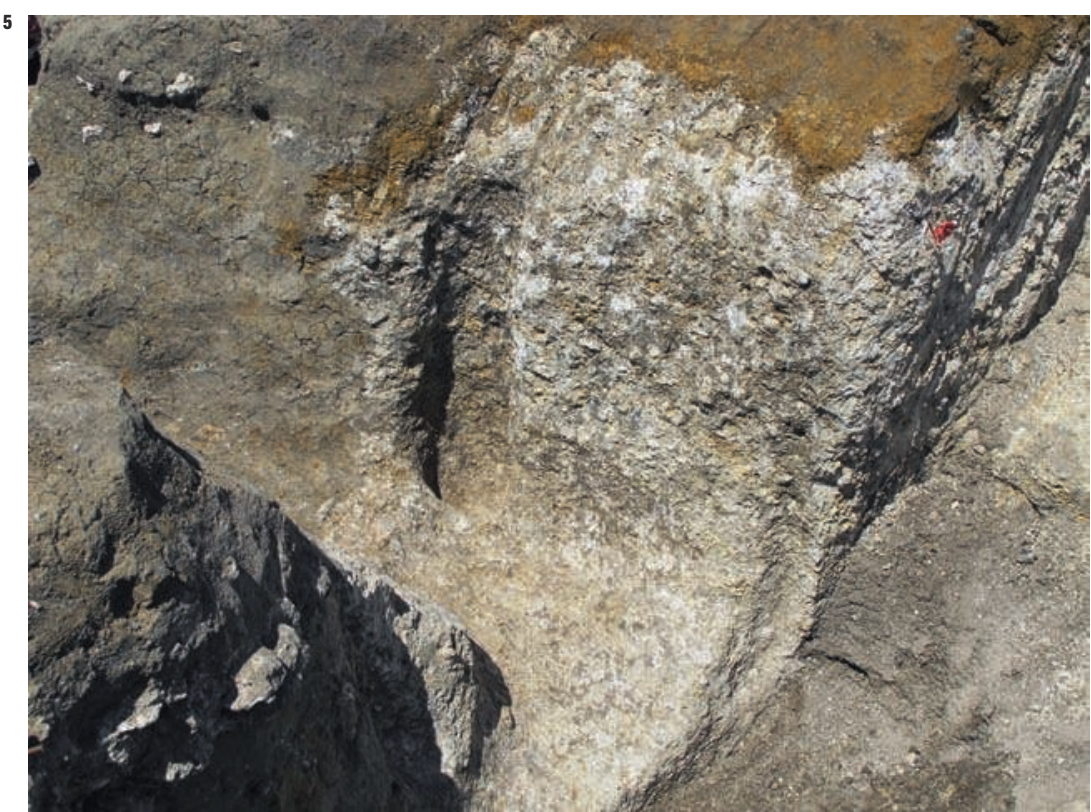


La première (pièce 2), de forme rectangulaire, est la plus vaste de l'ensemble. Elle mesure 3,30 $\mathrm{m}$ de long et 2,80 m de large avec une hauteur de voûte maximum de $1,90 \mathrm{~m}$. Une auge est ménagée dans une banquette laissée dans la paroi est du creusement. Le passage de la pièce 2 à la pièce 3 se fait au moyen d'une petite ouverture ovale large de $0,40 \mathrm{~m}$ et haute de $0,50 \mathrm{~m}$. Cette dernière pièce est de forme circulaire, son diamètre atteint $2 \mathrm{~m}$ environ et sa hauteur 1,10 m [ill. 6].

Le mode de construction du souterrain d'Ormes associe un creusement en tranchée et un accès en puits. Dans un premier temps, le puits (F.1-274) a été creusé puis, dans un deuxième temps, a été effectué l'excavation latérale d'un étroit passage et celle d'une unité souterraine, salle ou galerie. Simultanément, un autre accès a été aménagé à partir d'une tranchée de surface devenant, au fur et à mesure de son approfondissement, une galerie souterraine.

\section{Fonction et usage déduits des données archéozoologiques et physicochimiques}

Les souterrains médiévaux ont longtemps été considérés comme autant de systèmes défensifs attachés à une unité d'habitation ou à de plus vastes entités (mottes féodales, châteaux, abbaye, villages...). Ces souterrains refuges présentent un certain nombre de caractéristiques communes. Ils sont composés d'une ou plusieurs salles desservies par d'étroits couloirs coudés par tronçons rectilignes et généralement barrés de portes placées après un coude du couloir (Schweitz, 1971). Si, dans le cas du souterrain d'Ormes, le caractère défensif ne doit pas être totalement écarté, sa taille modeste et les éléments mis au jour lors de sa fouille semblent relever d'un autre type d'utilisation.

Ce sont au total 2465 restes osseux qui ont été mis au jour dans l'ensemble de la structure ${ }^{2}$ [ill. 7]. La composition du spectre faunique découvert dans l'accès F.1-3o et dans le silo (F.1-314) [ill. 8] témoigne de certaines particularités : présence de déchets alimentaires (bœuf, porc, âne) absents ailleurs. Les déchets alimentaires sont essentiellement représentés dans les couches de circulation, dans les remblais de colmatage de l'accès (F.1-30) et dans le comblement du silo (F.1-314). Ils sont majoritairement déposés après l'abandon de la structure daté, grâce au mobilier céramique, du courant du XIII ${ }^{\mathrm{e}} \mathrm{s}$. Les cadavres abandonnés, concentrés dans la pièce 2 , sont ceux de coqs, de chiens et de caprinés [ill. 9]. Ce constat orientait donc lidentification de cette structure souterraine comme une annexe de l'habitat, de type bergerie et poulailler. La fouille du souterrain a soulevé un certain nombre de questions, relatives principalement à l'usage des pièces qui le constituent. Pour apporter des éléments complémentaires à la réflexion archéologique, une série de prélèvements échantillonnés lors de la fouille ont fait l'objet d'une analyse pour certains éléments chimiques [cf. encadré p. 58]. Le C/N (rapport carbone sur azote) est en moyenne de 9, la matière organique est donc bien décomposée, cinq échantillons sont toutefois supérieurs à 10 sans excéder 11,22. Les taux de potassium total sont en moyenne de $3400 \mathrm{mg} / \mathrm{kg}$. Un groupe de prélèvements atteint des taux très élevés, autour de $4900 \mathrm{mg} / \mathrm{kg}$, également associés à des taux élevés du manganèse (corrélation linéaire entre les deux éléments, $r$ de o,95). Le potassium échangeable a aussi été mesuré. Il a tendance à suivre les taux de $P$ et est surtout corrélé au pourcentage de matière organique $(\mathrm{r}$ de 0,94$)$.

\section{Présentation des résultats par pièce}

Cinq prélèvements ont été analysés pour la pièce 1 . Ils présentent tous des résultats assez proches en termes de comportement physicochimique, excepté pour l'un d'eux. Les résultats de phosphore total, pour quatre d'entre eux, varient entre 6359 et 8925 ppm (faibles à moyens). Les phosphores organiques et les phosphores inorganiques restent faibles (avec une prédominance des derniers), ainsi que la matière organique et le potassium échangeable. En revanche, potassium et magnésium total sont élevés. Le rapport carbone sur phosphore, lui aussi, présente pour cette salle le plus fort taux de la série, sans que cela puisse s'expliquer (cependant, le résultat à 10,50 rappelle l'échantillon sur cailloux calcaires, non retenu comme référence).

Léchantillon qui sort du lot présente un $\mathrm{pH}$ moins basique $(7,87)$, associé à un très faible taux de phosphore total (1 700 ppm seulement, le plus faible de la série) et un rapport phosphores organiques/phosphores inorganiques élevé. Le résultat de ce dernier ainsi que les taux élevés du carbone et de l'azote, mais également de la matière organique et du potassium échangeable, laissent penser qu'il s'agit d'un prélèvement «pollué » par des apports récents (infiltration par le puits d'aération?). On notera néanmoins que les résultats du potassium total et du manganèse ne diffèrent pas des résultats des autres prélèvements.

Le comportement physicochimique de la mangeoire, F1.317, dans la pièce 2, rappelle celui de la pièce 1, mais avec des taux de Pt moyens et les taux de potassium total et de magnésium les plus élevés de la série. Le rapport carbone sur phosphore, là encore, oscille entre 9 et 10.

L'origine d'un potassium et d'un magnésium total très élevés, alors que le potassium échangeable et la matière organique sont très faibles, ne s'explique pas pour l'instant, dans l'état de nos connaissances du comportement de ces éléments.

Les taux de phosphore total dans la pièce 2 , sont là encore moyens, mais il apparaît que le phosphore organique augmente nettement (1 $582 \mathrm{ppm})$. Pourquoi une telle importance du phosphore organique? S'agit-il d'une pollution actuelle? En effet, le phosphore organique se minéralise avec le temps, expliquant l'importance générale sur un site archéologique du phosphore minéralisé, qui correspond au phosphore inorganique.
员 


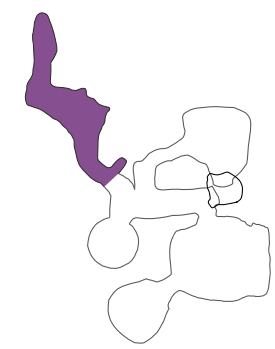

\begin{tabular}{|c|c|c|c|c|c|}
\hline F1-30 & NR & \%NR & PR & \%PR & PM \\
\hline boeuf & 5 & 7,4 & 52 & 18,6 & 10,4 \\
\hline porc & 17 & 25,0 & 60 & 21,5 & 3,5 \\
\hline caprinés & 24 & 35,3 & 98 & 35,1 & 4,1 \\
\hline âne & 1 & 1,5 & 20 & 7,2 & \\
\hline chat & 1 & 1,5 & 22 & 7,9 & \\
\hline coq & 19 & 27,9 & 26 & 9,3 & \\
\hline lézard vert & 1 & 1,5 & 1 & 0,4 & \\
\hline Déterminés & 68 & 80,0 & 279 & 89,7 & 4,1 \\
\hline Indéterminés & 17 & 20,0 & 32 & 10,3 & 1,9 \\
\hline TOTAL & $\mathbf{8 5}$ & $\mathbf{1 0 0}$ & $\mathbf{3 1 1}$ & $\mathbf{1 0 0}$ & $\mathbf{3 , 7}$ \\
\hline
\end{tabular}

18

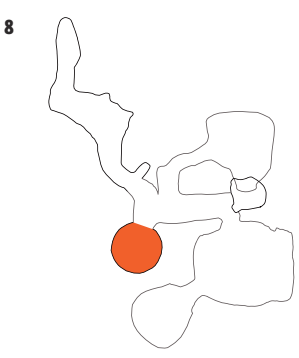

\begin{tabular}{|c|c|c|c|c|c|}
\hline F1-314 & NR & \%NR & PR & \%PR & PM \\
\hline bouf & 1 & 1,4 & 26 & 21,8 & 26 \\
\hline porc & 6 & 8,2 & 32 & 26,8 & 5,3 \\
\hline caprinés & 16 & 21,9 & 40 & 33,5 & 2,5 \\
\hline chien & 5 & 6,9 & 6 & 5 & 1,2 \\
\hline coq & 8 & 11 & 2 & 1,7 & \\
\hline oie & 2 & 2,7 & 14 & 11,7 & \\
\hline grive draine & 1 & 1,4 & 0 & 0 & \\
\hline grive, merle & 1 & 1,4 & 0 & 0 & \\
\hline rongeur & 7 & 9,6 & 0 & 0 & \\
\hline taupe & 20 & 27,4 & 0 & 0 & \\
\hline batracien & 6 & 8,2 & 0 & 0 & \\
\hline Déterminés & 73 & 81,1 & 119,5 & 93,7 & 1,6 \\
\hline Indéterminés & 17 & 18,9 & 8 & 6,3 & 0,5 \\
\hline TOTAL & $\mathbf{9 0}$ & $\mathbf{1 0 0}$ & $\mathbf{1 2 7 , 5}$ & $\mathbf{1 0 0}$ & $\mathbf{1 , 4}$ \\
\hline & & & & & \\
\hline
\end{tabular}

7. Tableau de

dénombrements

en nombre et poids

des restes de l'accès

au souterrain (F1-30).

Décompte des ossements

en nombre de restes mis

au jour dans l'accès de ce

souterrain. Des éléments

sont communs à l'ensemble

des contextes:

surreprésentation
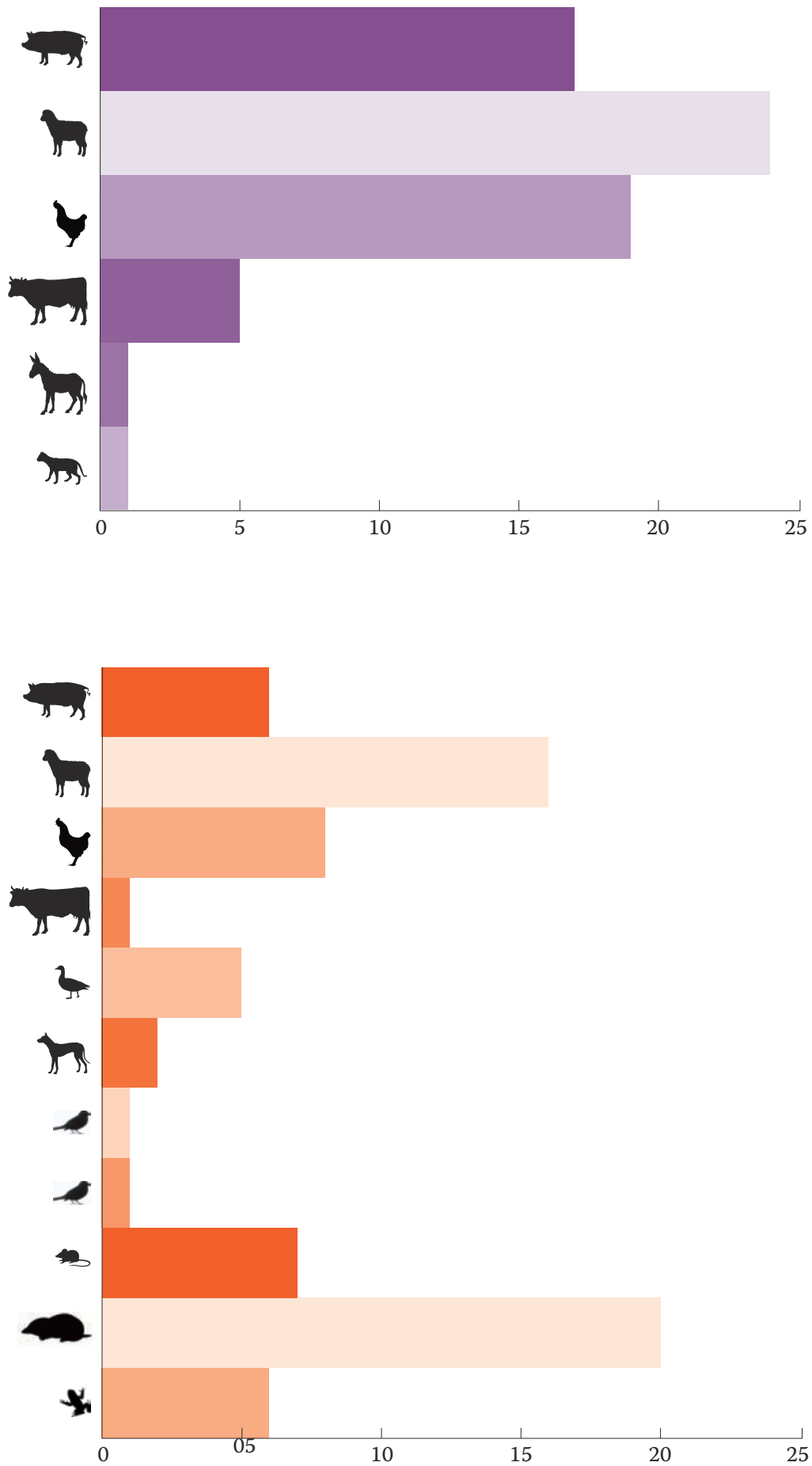

des coqs, des oies, des

chiens et des caprinés.

8. Tableau de

dénombrements

en nombre et poids des

restes du silo (F.1-314).

Décompte des ossements

en nombre de restes mis

au jour dans le silo (F.1-314). 


\begin{tabular}{|c|c|c|c|c|c|}
\hline F1-312 & NR & \%NR & PR & \%PR & PM \\
\hline porc & 2 & 0,7 & 4 & 10,1 & 2 \\
\hline caprinés & 67 & 24,5 & 21,5 & 54,4 & 0,3 \\
\hline chien & 79 & 28,9 & 5 & 12,7 & 0,1 \\
\hline coq & 74 & 27,1 & 10 & 25,3 & \\
\hline oie & 1 & 0,4 & 0 & 0 & \\
\hline passeriforme & 1 & 0,4 & 0 & 0 & \\
\hline rat noir & 1 & 0,4 & 0 & 0 & \\
\hline rongeur & 36 & 13,2 & 0 & 0 & \\
\hline taupe & 10 & 3,7 & 0 & 0 & \\
\hline batracien & 1 & 0,4 & 0 & 0 & \\
\hline escargot & 1 & 0,4 & 0 & 0 & \\
\hline Déterminés & 273 & 81 & 39,5 & 86,8 & 0,1 \\
\hline Indéterminés & 64 & 19 & 6 & 13,2 & 0,1 \\
\hline TOTAL & $\mathbf{3 3 7}$ & $\mathbf{1 0 0}$ & $\mathbf{4 5 , 5}$ & $\mathbf{1 0 0}$ & $\mathbf{0 , 1}$ \\
\hline & & & & & \\
\hline
\end{tabular}

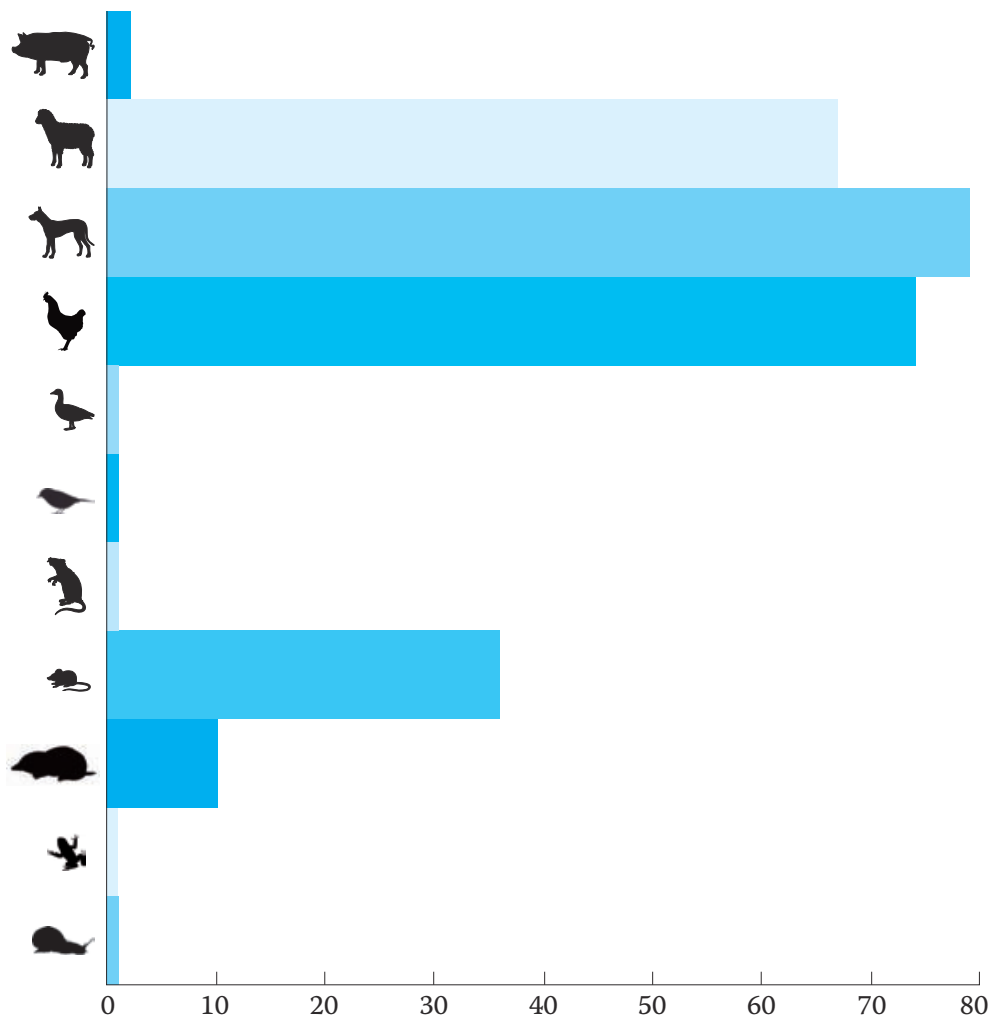

\section{Tableaude dénombrement} en nombre et poids des ossements animaux découverts dans la pièce 2 Décompte des ossements en nombre de restes mis au jour dans la pièce 2 (F.1-312). Les ossements de chiens, poules et caprinés sont majoritaires. Les déchets alimentaires sont très peu représentés (porc et oie). Les autres os (passeriforme, taupe, batracien, rongeur et rat noir) peuvent, comme dans le cas du silo (F.1-314), résulter de la présence des reliefs de repas de petits carnivores.
3. L'étude des traces d'outils tendrait à

conforter cette hypothèse d'un percement en deux points opposés de la cavité F.1-312 et du couloir

F.1-311. Toutefois, il faut rappeler les limite de

ce type d'observations, car rien ne nous indique qu'elles aient été réalisées lors de la phase

de percement initial des cavités : elles pourraient très bien résulter d'une phase de finition,

d'un réaménagemen

postérieur ou même de l'entretien de la pièce.
Cependant, une autre hypothèse est également plausible, celle de la présence ancienne de bois à cet endroit. Elle a été proposée pour d'autres sites et fait l'objet actuellement de publications (Liard, Froquet Uzel, à paraître). Néanmoins, en l'absence d'autres échantillons analysés dans la pièce, la possibilité d'une pollution par des matériaux actuels reste valable également.

Avec l'augmentation du phosphore inorganique, la matière organique et le potassium échangeable augmentent également un peu, alors que potassium et magnésium total sont moyens.

La petite salle adjacente à la pièce principale (pièce 3) apparaissait déjà un peu exceptionnelle en raison du type de sédimentation qui la caractérisait, mais elle s'avère également différente des autres pièces en termes physicochimiques puisqu'elle présente des taux de phosphore total élevés (17 ooo ppm), ainsi qu'une surreprésentation du carbone et de l'azote, même si le rapport carbone sur phosphore très bas confirme le processus de dégradation de la matière organique. Potassium et magnésium restent moyens, comme dans la pièce principale.

Une gradation des taux de phosphore enregistrés dans les pièces a été notée : les plus faibles sont dans la pièce 1 , ensuite ils augmentent dans la mangeoire et dans la pièce 2 , pour atteindre des taux élevés dans la pièce 3 . Il est vraisemblable que la pièce 2 , la mangeoire et la pièce 3 , tout particulièrement, aient contenu des résidus issus de fumiers ou de litières animales. C'est moins certain pour la pièce 1 , où des soupçons de pollution actuelle subsistent pour quelques prélèvements. Dans tous les cas, une réelle différenciation des espaces par leur comportement physicochimique s'observe : peut-elle s'expliquer par un usage différent? Dans les études actuelles sur des fumiers d'exploitations avicoles notamment, les taux diffèrent selon la fonction de l'espace : abreuvoir, mangeoire, dortoir (Balthazart, 2010, p. 43)... Il est possible qu'une telle distribution ait existé et se soit inscrite dans les principaux éléments que sont le carbone et l'azote ou encore le potassium et le magnésium, sinon le phosphore.

\section{Éléments d'interprétation}

Nous nous baserons, pour restituer le mode de construction du souterrain d'Ormes, sur les travaux de Patrice Conte et en particulier sur les hypothèses formulées lors de la fouille du souterrain de Beaulieu (Conte, 1990, p. 249-251).

La construction aurait débuté par le creusement de l'accès F.1-30 et, parallèlement, par le percement du puits F.1-274. Il est probable que ces deux excavations aient été reliées dans un premier temps par le seul couloir F.1-313 à partir duquel seront creusés le silo F.1-314, et les salles F.1-315 et F.1-316. Puis une seconde ouverture fut pratiquée dans la paroi du puits F.1-274 pour créer la salle F.1-312, le couloir F.1-311 étant sans doute réalisé à partir du couloir F.1-313 ${ }^{3}$. Au terme de la phase 
4. Interventions archéologiques préalables à la construction de l'autoroute A19 5. Nous ne repoussons pas l'idée qu'il ait pu être utilisé comme refuge temporaire. Cependant, cela ne semble pas avoir constitué sa destination première. d'excavation, les murets venant condamner le puits F.1-274 sont construits, le puits est rebouché et l'accès F.1-30=F.1-159 est couvert et fermé au moyen d'une porte ancrée dans F.1-318. Dans le souterrain, aucun aménagement du type d'une niche ou d'un trou de fixation ne semble avoir été réalisé pour accueillir un système d'éclairage. Pour les parties plus profondes du souterrain, ont été fouillés des espaces entre les maçonneries et la paroi du puits d'extraction. Cependant, nous n'avons pas pu observer de systèmes semblables à ceux décrits par Patrice Conte pour le souterrain de Chabalais (Haute-Vienne) : « un muret de pierres sèches ou plus rarement un dispositif de deux ou trois dalles de pierres verticales est aménagé à la base du puits avant son comblement depuis la surface. [...] La conception des conduits verticaux destinés, on le suppose, à l'aération des cavités, présente aussi des caractéristiques régulièrement attestées » (Conte, 1990, p. 251) [ill. 2-3] «Les nombreuses structures aménagées dans le substrat : alvéoles, rainurages, fosses au sol témoignent de systèmes de fermetures dans ce secteur » (Conte, Gauthier, 1985, p. 216-218). Seule la petite rainure mise au jour au niveau de la transition entre l'accès (F.1-30) et le couloir souterrain (F.1-313) peut apparaître comme le témoignage d'un système de fermeture.

Les souterrains médiévaux sont souvent considérés comme des systèmes défensifs attachés à une unité d'habitation ou à de plus vastes entités. L'entrée du souterrain refuge d'Aschères-le-
Marché, récemment fouillé ${ }^{4}$ dans l'est du Loiret, débouche dans une construction sur poteaux (Gilotte, 2008), tout comme le souterrain de «Beaulieu» (Conte, Gauthier, 1985), dont l'entrée débouche dans une maison en pierre. Les dimensions de ces constructions sont beaucoup plus importantes que celles du souterrain d'Ormes et leur caractère de refuge ne pose pas problème. Pour celui-ci, les sondages de la phase de diagnostic ont pu partiellement détruire les vestiges d'une construction installée sur l'entrée du souterrain. Seuls six trous de poteaux ont été mis au jour à proximité et ils ne permettent pas d'envisager la restitution d'un quelconque plan. En outre, les éléments de datation découverts en faible nombre ne permettent pas de s'assurer de leur contemporanéité.

C'est pourquoi, même si le caractère défensif ne doit pas être négligé, le souterrain d'Ormes, par sa taille modeste et les éléments mis au jour lors de sa fouille, nous semble relever d'un autre type d'utilisation ${ }^{\mathbf{5}}$. Outre le silo (F.1-314), les résultats des études archéozoologiques et physicochimiques s'accordent pour identifier cette structure souterraine comme une bergerie et un poulailler. On note en effet une forte représentation des squelettes complets ou non de caprinés, de chiots et de coqs. Cette surreprésentation va de pair avec la forte probabilité de dépôt de fumiers animaux dans une partie des cavités. Le cortège très complet des espèces représentées, tel le chat et le rat noir, nous indiquent que ce souterrain se trouve bien au sein d'un habitat et qu'il ne s'agit pas d'une dépendance agricole aménagée à l'écart.

\section{I'analyse physicochimique des sols archéologiques Morgane Liard. Inrap}

La mesure du phosphore constitue un indice de l'enrichissement d'origine anthropique des sols (Fidt, 1977 ; Schuldenrein, 1995 ; Holliday, 2004). Le phosphore se concentre là où la matière organique (tissus, os) a disparu et se conserve dans la plupart des sols à l'endroit de son dépôt avec des migrations verticales ou horizontales négligeables (Leonardi et al. 1999 ; Holliday, 2004, p. 304). Néanmoins, Goldberg et Macphail, exemples et citations à l'appui (Goldberg, Macphail, 2006, p. 347), rappellent que la bioturbation, ou encore l'illuviation, peuvent être à l'origine du transfert de phosphore dans les sols (selon des degrés variables en fonction de chaque site et du contexte physicochimique). Quant au carbone organique (C) et à l'azote total (N), ils renseignent sur la quantité de matières organiques dans les sols et permettent d'estimer leur dégradation (calcul du rapport $\mathrm{C} / \mathrm{N}$ ). Un $\mathrm{C} / \mathrm{N}$ bas (inférieur à 8-9) révèle une bonne décomposition, contre une mauvaise décomposition pour des valeurs supérieures à 9. Mais en contexte archéologique, ces échelles sont dépassées, la majorité des valeurs étant supérieures à 9 ; c'est sur l'amplitude des taux de C/N et leur importance relative que repose l'analyse. Ces mesures complètent celle du phosphore. Le rapport $\mathrm{C} / \mathbb{N}$ est influencé par les apports d'origine anthropique (matières carbonées, os, coquillages, etc.), au même titre que le pH. Le coefficient de corrélation $r$ de Pearson, appliqué à plusieurs séries de données, permet de mettre en évidence une relation linéaire entre deux éléments testés, mais elle n'est pas forcément causale.

Certains présentent une bonne corrélation entre eux, d'autres pas.

¥nfin, il faut préciser que les adjectifs «élevés ", « moyens », « faibles » et « très faibles », employés pour apprécier 1'importance des éléments, renvoient aux quatre classes de fréquences déterminées pour chacun d'eux. 


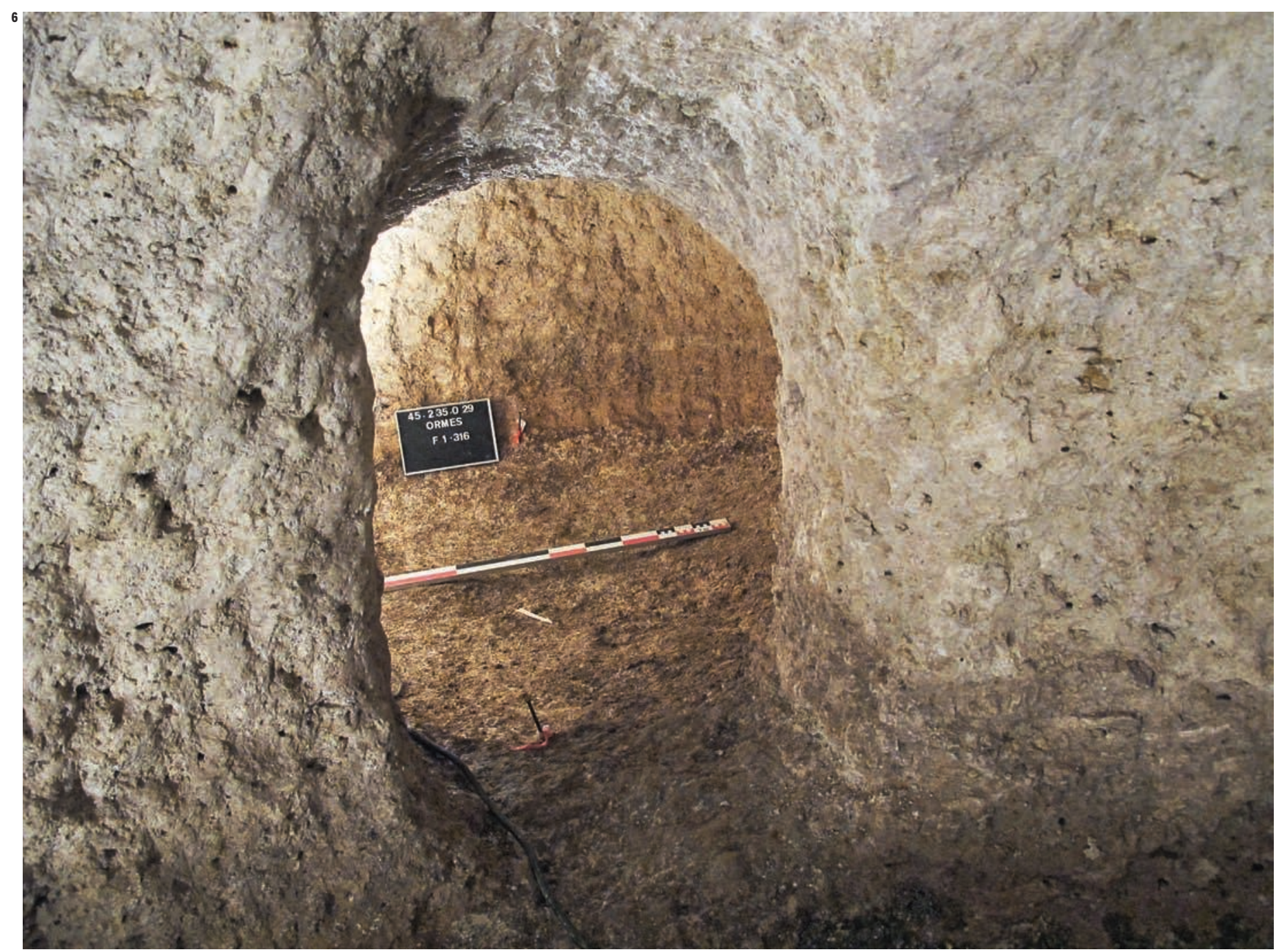

6. Vue du passage de

la pièce 2 à la pièce 3 .

\section{Références bibliographiques}

Baltazart A., 2010, Propriétés physiques, chimiques, biologiques et nutritives des litières en élevage de volailles, thèse de l'école vétérinaire d'Alfort, Faculté de médecine de Créteil, 184 p.

CONTE P. GAUTHIER F, 1985, «Beaulieu, site d'habitat du Moyen-Âge au Xx ${ }^{\mathrm{e}}$ siècle (Pensol, Haute-Vienne) ", Revue Archéologique du Centre de la France, t. 24, fasc. 2, p. 215-237.

CONTE P, 1990, «Souterrains, silos et habitat médiéval, état de la question archéologique en Limousin et Périgord », Heresis, 2, p. 243-281.

EIDT R.-C., 1977, « Detection and examination of anthrosols by phosphate analysis ", Science, vol. 197, $n^{\circ}$ 4311, p. 1327-1333.

Fournier L. (DIR.), 2011, Ormes, "Le Bois d'Ormes » (Loiret), Rapport Final d'Opération, Orléans, Inrap, SRA Centre, $707 \mathrm{p}$

GILOTTE S. (DIR.), 2008, Communes d'Aschères-le-Marché et Villereau (Loiret), «Le Marjolet » et «La Cabane », site A19-C-8 (n 45.009.023.AH), Rapport final d'opération, Inrap, SRA Centre, Orléans, p. 214-317.

Goldberg P., MACPHAIl R., 2006, Practical and theoretical geoarchaeology, Blackwell Science Ltd cop., 454 p.

HollidAy V.-T., 2004, Soils in Archaeological Research, Oxford University Press, 464 p.
LeONArdi G., Miglavacca M., Nardi S., 1999, « Soil phosphorus analysis as an integrative tool for recognizing buried ancient ploughsoils ", Journal of archaeological science, 26, p. 343-352.

LiARD M., Froquet-Uzel H., à paraître, « L'approche physicochimique, une possibilité de détection de la présence de bois : résultats de l'expérimentation sur quelques exemples de Courcelles (Loiret) datés de lâg du Bronze », in CARré F., Henrion F. (DIR.), Le bois dans l'architecture et l'aménagement de la tombe. Quelles approches?, Actes de la table-ronde d'Auxerre, 15-17 octobre 2009, t. XXII, Saint-Germain-en-Laye, AFAM.

Rivoire E., LiÉvaux N. (DIR.), 2009, Ormes « Le Bois d'Ormes, Lotissement », «Le Bois d'Ormes, Bassin de rétention » (Loiret-Centre), Rapport de diagnostic, Orléans, Inrap, SRA Centre, $236 \mathrm{p}$.

SCHULDENREIN J., 1995, « Geochemistry, phos bhate fractionation and the detection of activity areas at prehistoric north american sites », in CoLLINS M.-E. Carter B.-J., Gladfelter B.-G., Southard R.-J., Pedological perspectives in archaeological research Soil Science Society of America, special publication, $\mathrm{n}^{\circ}$ 4, p. 107-132.

SCHWEITZ D., 1971, « Cachettes et souterrains, refuges médiévaux », CVM-Cahiers médiévaux, 5 .

Consultable sur le site : http://www. cahiersmedievaux.com/CM5/refuges.htm 